\title{
Overexpression of CXCR4 promotes invasion and migration of non-small cell lung cancer via EGFR and MMP-9
}

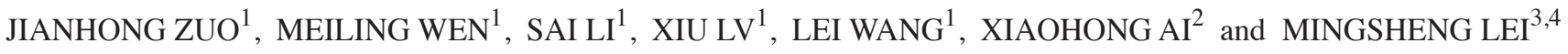 \\ ${ }^{1}$ Hunan Province Key Laboratory of Tumor Cellular and Molecular Pathology, \\ Cancer Research Institute, School of Medicine, University of South China, Hengyang; ${ }^{2}$ Department of Radiotherapy, \\ The First Affiliated Hospital of University of South China, Hengyang, Hunan 421001; ${ }^{3}$ Department of Respiratory, \\ Zhongnan Hospital of Wuhan University, Wuhan, Hubei 430071; ${ }^{4}$ Department of Respiratory and Critical Care Medicine, \\ The People's Hospital of Zhangjiajie City, Zhangjiajie, Hunan 427000, P.R. China
}

Received October 22, 2015; Accepted April 13, 2017

DOI: $10.3892 /$ ol.2017.7168

\begin{abstract}
The aim of the present study was to verify whether overexpression of CXC receptor 4 (CXCR4) promotes the invasion and migration of non-small cell lung cancer (NSCLC) via epidermal growth factor receptor (EGFR) and matrix metallopeptidase-9 (MMP-9), and to detect the association between CXCR4, EGFR and MMP-9. The effects of overexpression of CXCR4 on lung cancer cell functions were investigated by migration and invasion assays. Western blotting and zymograph assays were used to analyze the protein expression levels of EGFR and the production of MMP-9, respectively. Immunohistochemistry was applied to analyze the expression of EGFR, CXCR4 and MMP-9 in NSCLC. Statistical analyses were used to detect the associations among EGFR, CXCR4 and MMP-9 in NSCLC. Finally, survival analyses were performed. CXCR4 overexpression enhanced cell motility and invasion. CXCR4 also promoted expression of EGFR and elevated MMP-9 production. CXCR4, EGFR and MMP-9 were highly expressed in NSCLC, and were not identified as associated with age and sex $(\mathrm{P}>0.05)$. However, they were associated with tumor differentiation and lymph node metastasis $(\mathrm{P}<0.05)$. CXCR4, EGFR and CXCR4 expression were positively associated with one another in NSCLC $(\mathrm{P}<0.05)$. In addition, patients with positive expression of CXCR4, EGFR or MMP-9 in tumors exhibited significantly shorter overall survival compared with those with negative expression
\end{abstract}

Correspondence to: Professor Xiaohong Ai, Department of Radiotherapy, The First Affiliated Hospital of University of South China, 69 Chuanshan Road, Hengyang, Hunan 421001, P.R. China E-mail: 1163504926@qq.com

Dr Mingsheng Lei, Department of Respiratory and Critical Care Medicine, The People's Hospital of Zhangjiajie City, 192 Guyong road, Zhangjiajie, Hunan 427000, P.R. China

E-mail: 2846931246@qq.com

Key words: CXC receptor 4, epidermal growth factor receptor, matrix metallopeptidase 9 , non-small cell lung cancer
$(\mathrm{P}<0.05)$. In conclusion, $\mathrm{CXCR} 4$ overexpression enhanced cell motility and invasion via EGFR and MMP-9. CXCR4, EGFR and MMP-9 were identified as highly expressed in NSCLC, and there was positive correlation among them.

\section{Introduction}

Lung cancer is one of the most prominent causes of cancer-associated mortality in the world (1). Although the molecular network of lung carcinogenesis was partly understood, the high mortality rate has not markedly changed. Of the lung cancer cases diagnosed, $\sim 85 \%$ are non-small cell lung cancer (NSCLC), which has a poor prognosis and is difficult to treat (2). Despite years of research $(3,4)$, the survival rate of patients with NSCLC remains poor. Therefore, understanding the pathogenesis of lung cancer is important to those patients with NSCLC.

CXC receptor 4 (CXCR4), considered as the only receptor of stromal-derived-factor-1 (also termed CXCL12), is an $\alpha$-chemokine receptor (5). A number of studies have hypothesized that CXCR4 and CXCL12 increase the adhesive ability of tumors, as well as the degradation of the extracellular matrix (ECM) and basement membrane, which is beneficial to the invasion and metastasis of cancer cells (6-9). Kim et al (10) reported that LECs promote the migration of CXCR4-positive tumor cells by secretion of CXCL12. In turn, previous studies demonstrated that vascular endothelial growth factor increases the CXCR4 expression of endothelial cells, increases CXCL12 endothelial cell activity, promotes the generation of blood and lymphatic vessels and induces tumor cells to specific organs $(11,12)$. Epidermal growth factor receptor (EGFR), a type of transmembrane glycoprotein, exists on the cell surface and is encoded by the proto-oncogene c-erb-B1 (13). Ligand-mediated EGFR signaling, such as the RAS/extracellular-signal-regulated kinase (ERK) and phosphoinositide 3-kinase (PI3K)/protein kinase B pathways, regulates various cellular processes, including cell survival, death, growth, proliferation and motility (14). T798M mutations lead to EGFR overexpression or over-activity in breast cancer (15). Patients with NSCLC who exhibit co-expression of CXCR4 and EGFR achieve a more aggressive clinical progression of cancer (16). 
Furthermore, studies have revealed that two receptors, CXCR4 and EGFR, can enhance the capacity of cell chemotaxis and increase the invasion and metastatic ability of tumors $(16,17)$. However, the molecular mechanism by which CXCR4 and EGFR promote invasion and migration remains unclear.

Invasion and metastasis are the main characteristics of tumors and are responsible for the poor prognosis of advanced NSCLC, which involves multiple processes, including the downregulation of adhesion between cells and the ECM and degradation of the ECM, leading to invasion of new tissues and finally resulting in colonization (18). Matrix metalloproteinases (MMPs) are a large family of zinc-dependent peptidases, which perform important roles in tumor metastasis by degradation of the ECM proteins (19). MMP-9 is one of the important MMPs highly expressed in lung tumor cells, and its expression is associated with invasiveness, tumor growth and angiogenesis $(19,20)$. MMP-9 expression increases in the tissues of various malignant cancers, including lung, breast and head and neck cancers (21-23). Positive immunostaining of MMP-9 in NSCLC has an independent prognostic value for the diagnosis of distant metastasis or local recurrence (24). The present study aimed to detect the association between CXCR4 and MMP-9. The objective of the present study was to verify whether overexpression of CXCR4 promotes invasion and migration of NSCLC via EGFR and matrix metallopeptidase 9 (MMP-9) and to detect any association between CXCR4, EGFR and MMP-9.

\section{Materials and methods}

Antibodies. Monoclonal antibodies against the following were used: CXCR4 (catalog no. ab1760; Abcam, Cambridge, UK); EGFR (catalog no. sc-3049; Santa Cruz Biotechnology, Inc., Dallas, TX, USA); $\beta$-tubulin (catalog no. 560340; BD Pharmingen; BD Biosciences, San Jose, CA, USA); and MMP-9 antibody (5G3; catalog no. ab119906; Abcam). All the antibodies were diluted at 1:1,000. Anti-mouse (catalog no. sc-2371; Santa Cruz Biotechnology, Inc., Dallas, TX, USA) and anti-rabbit secondary antibodies (catalog no. sc-516087; Santa Cruz Biotechnology, Inc.), conjugated to horseradish peroxidase for immunoblotting or conjugated to fluorescein isothiocyanate, were obtained from Jackson ImmunoResearch Laboratories, Inc., West Grove, PA, USA.

Cell culture and transfection. The human lung adenocarcinoma A549 cell line (catalog no. 86012804; Sigma-Aldrich; Merck KGaA, Darmstadt, Germany) was cultured at $37^{\circ} \mathrm{C}$ in a humidified atmosphere of $5 \% \mathrm{CO}_{2}$. Cells were maintained in RPMI-1640 (Gibco; Thermo Fisher Scientific, Inc., Waltham, MA, USA) with $10 \%$ fetal bovine serum (Gibco; Thermo Fisher Scientific, Inc., Waltham, MA, USA), $1 \%$ penicillin and streptomycin (Gibco; Thermo Fisher Scientific, Inc.). Briefly, for constitutive expression of CXCR4, 5x10 A549 cells were transfected using Lipofectamine ${ }^{\circledR} 2000$ reagent, with CXCR4 in a pcDNA3 vector (catalog no. 13031; Addgene, Inc., Cambridge, MA, USA), and the stably transfected cells were selected with G418 to yield the CXCR4 cell line. Similarly, the mock-transfected cell line was generated from A549 cells using an empty vector.
For RNA interference analysis, the sequences of the small interfering RNAs (siRNAs) were as follows: CXCR4 siRNA sense, 5'-CCGACCUCCUCUUUGUCAUTT-3'; negative control siRNA sense, 5'-UUCUCCGAACGUGUCACGUTT-3'. siRNA targeting human CXCR4 was delivered into CXCR4 cells using Lipofectamine ${ }^{\circledR} 2000$ reagent (Invitrogen; Thermo Fisher Scientific, Inc.) according to the manufacturer's protocol. In addition, the non-targeting (scramble) siRNA pool was used at the same concentration (100 $\mathrm{nM})$ as a control for the RNA interference assays. At $48 \mathrm{~h}$ post-transfection $\left(37^{\circ} \mathrm{C}\right)$, cells were subjected to migration and invasion assays as described below or cell lysates were collected and analyzed by western blot analysis.

Migration and invasion assays. In the Transwell migration assay, as previously described $(21,25)$, the underside of the Transwell insert (pore size, $8 \mu \mathrm{m}$; Costar; Corning Incorporated, Corning, NY, USA) was pre-coated with collagen type I (Col-I, $1 \mu \mathrm{g} / \mathrm{ml})$ or fibronectin $(10 \mu \mathrm{g} / \mathrm{ml})$. Subsequently, 2x10 A549 and CXCR4 cells were loaded onto the upper chamber of the Transwell and the lower chamber was filled with serum-free medium. Cells were incubated for $4 \mathrm{~h}$ at $37^{\circ} \mathrm{C}$, fixed with $4 \%$ paraformaldehyde for $10-15 \mathrm{~min}$ at room temperature and stained with $0.1 \%$ crystal violet for $30 \mathrm{~min}$ at $37^{\circ} \mathrm{C}$. Non-migrating cells retained on the upper side were removed by wiping with a cotton swab. Cells that had migrated through the filter were counted and averaged from three randomly selected optical microscopic fields (20X objective).

In vitro invasion assays were performed using Transwell inserts (Corning Incorporated), which consisted of cell culture inserts containing $8 \mu \mathrm{m}$ pore size filters. Briefly, the Transwell inserts were coated with Matrigel $(1 \mathrm{mg} / \mathrm{ml}$; BD Biosciences) or Col-I (1.2 mg/ml, Sigma-Aldrich; Merck $\mathrm{KGaA}$, catalog no. SAB4200678), and allowed to gel at $37^{\circ} \mathrm{C}$ for $1 \mathrm{~h}$. Subsequently, A549 cells $\left(3 \times 10^{5}\right)$ in $200 \mu$ l serum-free Dulbecco's modified Eagle's medium (Biowest, Nuaillé, France) were added to the top of the Transwell. Serum-free medium was then added to the lower chamber and incubated for $24 \mathrm{~h}$ at $37^{\circ} \mathrm{C}$. Cells were fixed and stained with $0.1 \%$ crystal violet as previously. Matrigel and associated cells were removed with a cotton swab. Cells that had penetrated the Matrigel and had reached the underside of the filter membrane were then counted and averaged from 3 randomly selected microscopic fields with an optical microscope (magnification, $\mathrm{x} 200)$.

Western blotting. The conditioned medium without FBS was concentrated in a $50 \mathrm{ml}$ centrifuge tube. Cells $\left(10^{6}\right)$ were extracted using lysis buffer [50 mmol/1 Tris ( $\mathrm{pH} 7.5)$, $500 \mathrm{mmol} / 1 \mathrm{NaCl}, 1 \%$ Triton $\mathrm{X}-100,0.5 \%$ sodium deoxycholate, $0.1 \%$ SDS, $10 \mathrm{mmol} / 1 \mathrm{MgCl} 2$ and complete protease inhibitor mixture (Roche Molecular Diagnostics, Pleasanton, CA, USA)]. Protein concentrations were determined with the bicinchoninic acid protein assay kit (Pierce; Thermo Fisher Scientific, Inc.) according to the manufacturer's protocol. Protein (10 $\mu \mathrm{g}$ per lane) was separated using 10\% SDS-PAGE. Following transferal onto nitrocellulose membranes (EMD Millipore, Billerica, MA, USA). Non-specific binding was blocked by the $5 \%$ skimmed milk solution at $37^{\circ} \mathrm{C}$ for $45 \mathrm{~min}$. 
Then proteins were probed with primary antibodies against CXCR4, EGFR or $\beta$-tubulin overnight at $4^{\circ} \mathrm{C}$ and secondary horseradish peroxidase-coupled antibodies at room temperature for $45 \mathrm{~min}$. Following 3 washes with phosphate-buffered saline, blots were developed using an enhanced chemiluminescence system (GE Healthcare Life Sciences, Chalfont, UK).

Gelatinolytic zymography. Gelatinolytic zymography of MMP-9 activity was performed using SDS-PAGE (7.5\% gel) containing $2.56 \mathrm{mg} / \mathrm{ml}$ gelatin. A mix of one part sample with one part Tris-glycine SDS 2X Sample Buffer was left to stand for $10 \mathrm{~min}$ at room temperature. Gels were run until completion, indicated by the bromophenol blue tracking dye reaching the bottom of the gel, incubated in the zymogram renaturing buffer [2.5\% Triton X-100, $50 \mathrm{mmol} / 1$ Tris- $\mathrm{HCl}, 5 \mathrm{mmol} / \mathrm{l}$ $\mathrm{CaCl}_{2}$ (pH 7.6)]. Subsequently, gentle agitation for $30 \mathrm{~min}$ at room temperature was performed and the renaturing buffer was replaced with $1 \mathrm{X}$ zymogram developing buffer $[0.5 \mathrm{M}$ Tris- $\mathrm{HCl}$ (pH 7.45), 2.0 M NaCl, $50 \mathrm{mM} \mathrm{CaCl}_{2}, 0.2 \%$ Brij-35]. Gels were gently agitated for $30 \mathrm{~min}$ at room temperature and fresh $1 \mathrm{X}$ zymogram developing buffer was added prior to incubation at $37^{\circ} \mathrm{C}$ for $4 \mathrm{~h}$. Staining was performed using $0.5 \%$ (w/v) Coomassie Blue R-250 (Beijing Solarbio Science \& Technology Co., Ltd., Beijing, China) for $30 \mathrm{~min}$. Gels were destained with an appropriate Coomassie Blue R-250 destaining solution (methanol, acetic acid and water, 50:10:40). Areas of protease activity appeared as clear bands against a dark blue background where the protease had digested the substrate.

Patients and specimens. Tissue from surgery was collected from 61 patients from the People's Hospital of Zhangjiajie City (Zhangjiajie, China), who were confirmed by pathological diagnosis of lung cancer without radiotherapy and chemotherapy treatment. The study was approved by the ethics committee of the People's Hospital of Zhangjiajie City and patients provided written informed consent. The study included 36 males and 25 females, whereby 41 individuals were $\geq 60$ years old and 20 individuals were $<60$ years old. Of these patients, according to the classification standard of the World Health Organization of lung cancer tumor histological grade (26), 16 cases of poorly differentiated carcinoma were identified, as well as 45 cases of well/moderately differentiated carcinoma. Following surgery, the tissues were formalin-fixed and paraffin-embedded. The characteristics of the patients are summarized in Table I.

$H \& E$ staining and immunohistochemical technique. The lesions were visualized with hematoxylin and eosin (H\&E) staining. Immunohistochemical analysis for CXCR4, EGFR and MMP-9 was performed with 5- $\mu \mathrm{m}$ formalin-fixed, paraffin-embedded tissue sections using a standard immunohistochemical technique as previously described (27).

Survival analysis. The duration of overall survival was calculated from the date of first diagnosis of the disease to the date of mortality or the last follow-up. The Kaplan-Meier method was used for survival analysis, and Cox proportional hazards models were used to evaluate the association between the expression of CXCR4, EGFR and MMP-9 and the prognosis of patients with NSCLC. Significant prognostic variables, including patient age, tumor stage and type of treatment were included in these models. Cox regression plots were constructed for $\mathrm{CXCR}^{+}$vs. $\mathrm{CXCR}^{-}, \mathrm{EGFR}^{+}$vs. EGFR ${ }^{-}$and

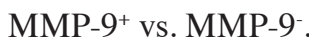

Statistical analysis. SPSS 19.0 statistical software was used for the analysis of the image data (IBM SPSS, Armonk, NY, USA). The data were presented as the mean \pm standard deviation. The $\chi^{2}$ test was applied to analyze the association between CXCR4, EGFR and MMP-9 and the clinical features of NSCLC. Spearman's rank correlation analysis was applied to detect the correlation among CXCR4, EGFR and MMP-9. $\mathrm{P}<0.05$ was considered to indicate a statistically significant difference.

\section{Results}

CXCR4 overexpression enhances cell motility and invasion. To investigate the role of CXCR4 in invasiveness and metastatic potential, CXCR4 was overexpressed in the lung cancer A549 cell line, which normally expresses background levels of CXCR4 protein (28). The CXCR4 cell line represents a stable population of cells derived by transfection with an experimental plasmid (Fig. 1A). The migration and invasion ability of CXCR4-overexpressing cells was evaluated, and it was identified that CXCR4 cells migrated faster than the parental or mock controls on Col-I (Fig. 1B) and fibronectin substrates (Fig. 1C) in Transwell assays. The invasive capacity of the cell line through the ECM barrier was then examined. The results demonstrated that CXCR4 cells obtained a marked increase in invasion compared with control cells towards Matrigel (Fig. 1D) and Col-I (Fig. 1E). Together, these data indicated that CXCR4 expression enhanced motility and invasive ability of lung carcinoma cells.

CXCR4 promotes expression of EGFR and elevates MMP-9 production. To define the mechanism of CXCR4-enhanced cell motility, critical proteins known to regulate cell migration and invasion were examined. EGFR was identified to be increased in CXCR4 cells compared with controls (Fig. 2A). The constitutive expression of EGFR in metastatic tumor cells has previously been demonstrated to be associated with enhanced MMP-9 expression and induction of invasive capacity (25). In the present study, it was observed that CXCR4-overexpressing cells elevated MMP-9 production compared with controls (Fig. 2B). To verify the aforementioned results, the effect of CXCR4 knockdown was analyzed by targeting with specific siRNA. The siRNA was efficient in reducing CXCR4 compared with the scramble oligonucleotide (the non-targeting siRNA) (Fig. 3A). Having confirmed the CXCR4 knockdown, the expression of EGFR and MMP-9 production and the migration and invasion ability of these cells was measured. The data demonstrated that CXCR4 knockdown inhibited the expression of EFGR (Fig. 3A). At the same time, knockdown of CXCR4 was identified to markedly attenuate MMP-9 production compared with controls (Fig. 3B). It was also detected that the number of migrating cells following CXCR4 suppression was significantly decreased compared with that of the untreated cells (Fig. 3C and D). Cell invasion assays with Matrigel barriers 
Table I. Summary of baseline patient characteristics $(n=61)$.

\begin{tabular}{lc}
\hline Parameter & Value \\
\hline Age & \\
Range, years & $41-82$ \\
Median, years & 61.1 \\
$<60, \mathrm{n}(\%)$ & $20(33)$ \\
$\geq 60, \mathrm{n}(\%)$ & $41(67)$ \\
Sex, n $(\%)$ & \\
Male & $36(59)$ \\
Female & $25(41)$ \\
Tumor differentiation, $\mathrm{n}(\%)$ & \\
Well/moderate & $45(74)$ \\
Poor & $16(26)$ \\
Lymph node metastasis, $\mathrm{n}(\%)$ & $31(51)$ \\
No & $30(49)$ \\
Yes & \\
\hline
\end{tabular}

and Col-I also demonstrated that suppression of CXCR4 expression significantly reduced the number of invading cells (Fig. 3E and F).

CXCR4, EGFR and MMP-9 are highly expressed in NSCLC. A total of 61 patients (36 males and 25 females) were included in the case series. The characteristics of the patients are summarized in Table I. The expression of CXCR4 and EGFR was analyzed by immunohistochemistry in clinical specimens of lung cancer and normal lung tissue. The expression levels of CXCR4, EGFR and MMP-9 were identified to be higher in NSCLC tissues compared with normal lung tissue (Fig. 4). Out of the total 61 specimens, the rates of positive expression of CXCR4, EGFR and MMP-9 in NSCLC were 69, 76 and $64 \%$, respectively. CXCR4, EGFR and MMP-9 expression in tissues of patients with NSCLC was not associated age and sex ( $P>0.05)$, but was associated with tumor differentiation and lymph node metastasis $(\mathrm{P}<0.05$; Table II).

EGFR, CXCR4 and MMP-9 are positively correlated in $N S C L C$. Using Spearman's rank correlation analysis, significant association between EGFR and CXCR4 expression was observed ( $\mathrm{r}=0.422$; $\mathrm{P}<0.05$; Table III). The expression of CXCR4 and MMP-9 in the NSCLC group demonstrated positive correlation $(\mathrm{r}=0.438 ; \mathrm{P}<0.05$; Table IV), and the expression of EGFR was also positively correlated with MMP-9 ( $r=0.328$; $\mathrm{P}<0.05$; Table V).

Survival analysis. The median survival time for cases with $\mathrm{CXCR}^{+}{ }^{+}$was 10.0 months [95\% confidence (CI), 8.308-11.692] and 22.0 months for patients with CXCR4- $(95 \% \mathrm{CI}$, 14.133-29.867; $\mathrm{P}<0.05$; Fig. 5A). The median survival time for patients with $\mathrm{EGFR}^{+}$was 10.0 months $(95 \%$ CI, 7.947-12.053) and 20.0 months for patients with EGFR $(95 \% \mathrm{CI}$, 11.938-28.062; P<0.05; Fig.5B). Patients with MMP-9+ tumors (median survival time, 12.0 months; 95\% CI, 10.576-16.120) had significantly shorter overall survival times compared
A

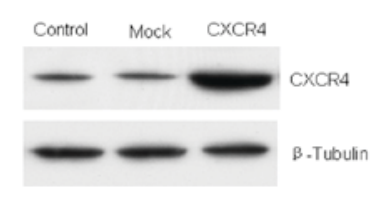

B
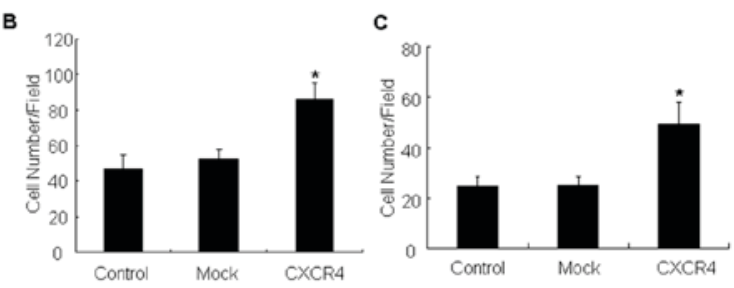

D

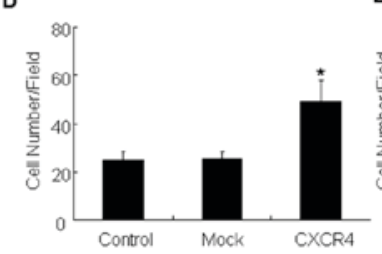

E.

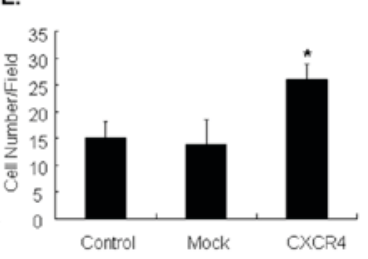

Figure 1. CXCR4 enhances tumor cell invasion and metastasis. (A) CXCR4 expression in control, mock and CXCR4-transfected cells was verified by western blotting with antibodies to CXCR4. Tubulin was used as a loading control. Control, mock and CXCR4-expressing cells were subjected to $4 \mathrm{~h}$ migration assays using Transwells coated with (B) Col-I or (C) fibronectin substrate. The number of cells that migrated through the Transwell membranes was estimated by counting at least three random microscopic fields. Results are the mean \pm standard deviation $\left({ }^{*} \mathrm{P}<0.05\right)$. Cell invasion assays were performed for $24 \mathrm{~h}$ on Transwells coated with (D) Matrigel or (E) Col-I. The number of cells that migrated through the Transwell membranes was determined by counting at least three randomly microscopic fields. Results are the mean \pm standard deviation $\left({ }^{*} \mathrm{P}<0.05\right)$. CXCR4, CXC receptor 4; SD, standard deviation; Col-I, collagen type I.

with patients with MMP-9 tumors (median survival time, 26.0 months; 95\% CI, 21.124-32.641; P<0.05; Fig. 5C).

\section{Discussion}

Lung cancer with high incidence and mortality is the leading cause of cancer-associated mortality in the western world, and the 5-year survival rate of NSCLC is estimated at $\sim 15 \%$ (29). In addition to surgery, chemotherapy is a crucial element of treatment for patients with NSCLC (30). Therefore, an emerging understanding of the molecular pathways that characterize cell growth, cell cycle, apoptosis, angiogenesis and invasion is of crucial importance to the treatment of patients with lung cancer. Previous studies reported that treating leukemia with a combination of CXCR4 inhibitors and chemotherapeutic agents produced additive therapeutic effects $(31,32)$. Overexpression of EGFR is common in head and neck squamous cell carcinoma (HNSCC) (33). Previous data have presented EGFR as a new target for anti-HNSCC therapy (34). However, the mechanism of CXCR4 and EGFR-stimulated tumor cell invasion and migration requires to be elucidated.

CXCR4, a seven transmembrane $\mathrm{G}$ protein-coupled receptor, is widely expressed in various cell types, including endothelial, epithelial and hematopoietic stem cells, lymphocytes and cancer cells (35). Previous studies indicated that 
A

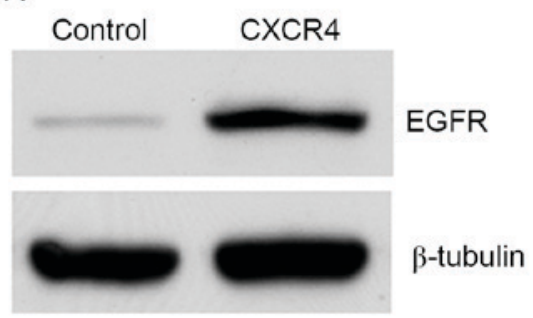

B

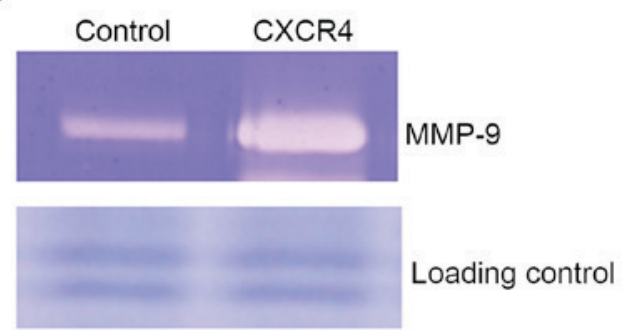

Figure 2. CXCR4 modulates EGFR protein expression and MMP-9 production. (A) EGFR expression was analyzed by western blot analysis of control, mock and CXCR4-expressing cells. $\beta$-tubulin was used as a loading control. (B) A representative result of gelatinolytic zymography demonstrated MMP-9 activity in the conditioned medium from control and CXCR4-expressing cells. Loading control, non-target protein bands in Coomassie Blue staining gel prior to transferring to a nitrocellulose membrane. CXCR4, CXC receptor 4; EGFR, epidermal growth factor 4; MMP-9, matrix metallopeptidase-9.

A

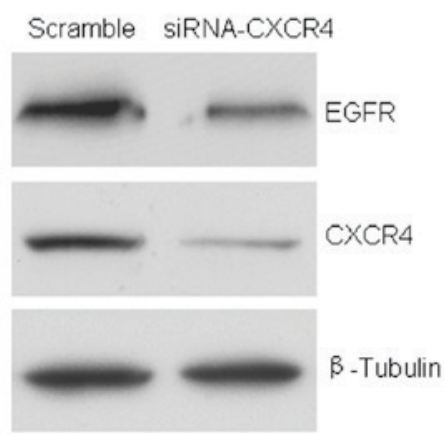

C

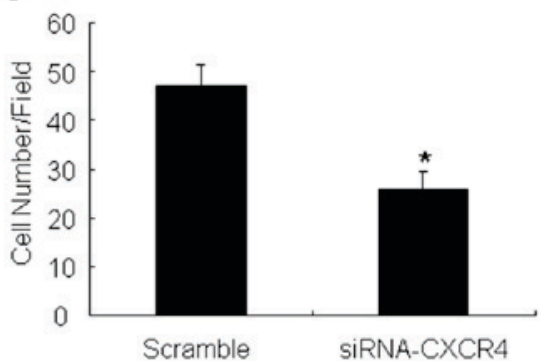

$\mathbf{E}$

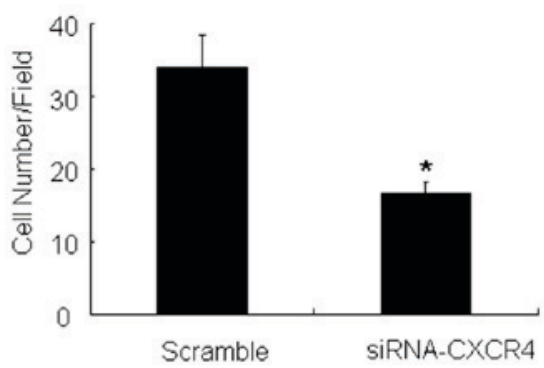

B

Scramble siRNA-CXCR4

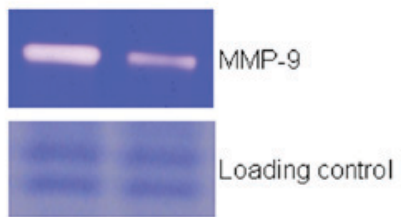

D

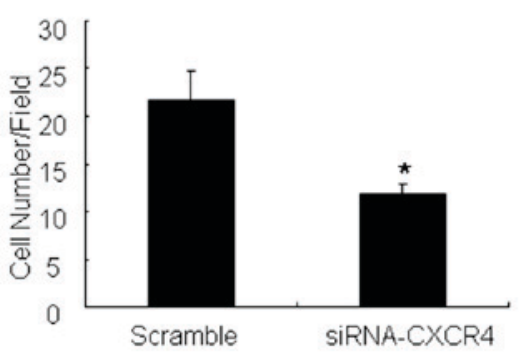

$\mathbf{F}$

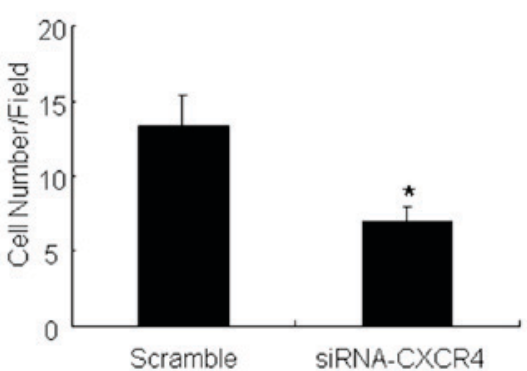

Figure 3. CXCR4 knockdown inhibits cell migration and invasion induced by EGFR expression in A549 cells. (A) Western blot analysis demonstrated the expression levels of CXCR4 and EGFR in A549 cells transfected with scramble or CXCR4 siRNA. (B) Conditioned medium was prepared from A549 cells transfected with scramble or CXCR4 siRNA, and MMP-9 activity was detected by gelatinolytic zymography. Loading control, non-target protein bands in Coomassie Blue staining gel prior to transferal onto a nitrocellulose membrane. A549 cells transfected with scramble or CXCR4 siRNA were subjected to a Transwell migration assay with (C) Col-I or (D) fibronectin. Results are the mean \pm standard deviation ( $\mathrm{P}<0.05)$. A549 cells transfected with scramble or CXCR4 siRNA were subjected to a Transwell invasion assay on Transwells coated with (E) Matrigel or (F) Col-I. Results are the mean \pm standard deviation ( $\mathrm{P}<0.05)$. CXCR4, CXC receptor 4; EGFR, epidermal growth factor 4; MMP-9, matrix metallopeptidase-9; siRNA, small interfering RNA; Col-I, collagen type I; SD, standard deviation.

CXCR4 is involved in cellular proliferation, migration and metastasis of solid tumors in a variety of cancers, including breast, colorectal and gastric cancer (36-38). A previous study has demonstrated that the CXCL12/CXCR4 axis is involved in tumor progression, angiogenesis, metastasis and survival (38). In the present study, the results demonstrated that CXCR4 is 
Table II. Association between EGFR, CXCR4 and MMP-9 expression and clinicopathological factors in NSCLC.

\begin{tabular}{|c|c|c|c|c|c|c|c|c|c|}
\hline \multirow[b]{2}{*}{ Parameter } & \multicolumn{2}{|c|}{ CXCR4, n (\%) } & \multirow[b]{2}{*}{ P-value } & \multicolumn{2}{|c|}{ EGFR, n (\%) } & \multirow[b]{2}{*}{ P-value } & \multicolumn{2}{|c|}{ MMP-9, n (\%) } & \multirow[b]{2}{*}{ P-value } \\
\hline & Low & High & & Low & High & & Low & High & \\
\hline Age, years & & & 0.142 & & & 0.537 & & & 1 \\
\hline$<60$ & $10(53)$ & $27(64)$ & & $6(40)$ & $14(30)$ & & $7(32)$ & $13(33)$ & \\
\hline$\geq 60$ & $9(47)$ & $15(36)$ & & $9(60)$ & $32(70)$ & & $15(68)$ & $26(67)$ & \\
\hline Sex & & & 0.266 & & & 0.13 & & & 0.416 \\
\hline Male & $8(42)$ & $28(67)$ & & $6(40)$ & $30(76)$ & & $11(50)$ & $25(64)$ & \\
\hline Female & $11(68)$ & $14(33)$ & & $9(60)$ & $16(24)$ & & $11(50)$ & $14(36)$ & \\
\hline Tumor differentiation & & & 0.025 & & & 0.015 & & & 0.033 \\
\hline Well & $10(42)$ & $35(64)$ & & $7(47)$ & $38(61)$ & & $20(91)$ & $25(64)$ & \\
\hline Poor & $9(58)$ & $7(36)$ & & $8(63)$ & $8(39)$ & & $2(9)$ & $14(36)$ & \\
\hline Lymph node metastasis & & & 0.002 & & & 0.013 & & & 0.008 \\
\hline Yes & $4(21)$ & $27(64)$ & & $5(26)$ & $26(62)$ & & $16(73)$ & $14(36)$ & \\
\hline No & $15(79)$ & $15(36)$ & & $14(74)$ & $16(38)$ & & $6(27)$ & $25(64)$ & \\
\hline
\end{tabular}

P-values were calculated using the $\chi^{2}$ test. EGFR, epidermal growth factor receptor; CXCR4, CXC receptor 4; MMP-9, matrix metallopeptidase-9.

Table III. Spearman's rank correlation analysis of clinical association between CXCR4 and EGFR.

\begin{tabular}{lccc}
\hline & & \multicolumn{2}{c}{ EGFR } \\
\cline { 3 - 4 } & Expression & Low & High \\
\hline \multirow{2}{*}{ CXCR4 } & Low & 8 & 11 \\
& High & 9 & 33 \\
\hline
\end{tabular}

$\mathrm{r}=0.214 ; \mathrm{P}<0.05 . \mathrm{CXCR} 4, \mathrm{CXC}$ receptor 4 . EGFR, epidermal growth factor receptor.

Table IV. Spearman's rank correlation analysis of clinical association between CXCR4 and MMP-9.

MMP-9

\begin{tabular}{lcrc} 
& Expression & Low & High \\
\cline { 3 - 4 } CXCR4 & Low & 12 & 10 \\
& High & 4 & 35 \\
\hline
\end{tabular}

r=0.476; $\mathrm{P}<0.001 . \mathrm{CXCR} 4, \mathrm{CXC}$ receptor 4; MMP-9, matrix metallopeptidase- 9 .

highly expressed in NSCLC and promotes cellular migration and invasion, which is consistent with the aforementioned studies.

EGFR, is a transmembrane glycoprotein and can bind to ligands, including transforming growth factor $\alpha$ and EGF, with
Table V. Spearman's rank correlation analysis of clinical association between EGFR and MMP-9.

\begin{tabular}{lcrc}
\hline & & \multicolumn{2}{c}{ MMP-9 } \\
\cline { 3 - 4 } & Expression & Low & High \\
\hline \multirow{2}{*}{ EGFR } & Low & 10 & 12 \\
& High & 6 & 33 \\
\hline
\end{tabular}

$\mathrm{r}=0.328 ; \mathrm{P}<0.05$. EGFR, epidermal growth factor receptor; MMP-9, matrix metallopeptidase-9.

its extracellular domains to promote receptor dimerization and activation of a cytoplasmic tyrosine kinase domain (39). The involvement of EGFR signaling has been demonstrated in various cellular processes ranging from normal growth and differentiation to oncogenesis (40-42). Aberrant EGFR as a result of receptor mutation and/or overexpression occurs in NSCLC (43) laryngeal cancer (44), pancreatic cancer (45). Our previous study indicated that EGFR activation promotes HNSCC cell migration and invasion by inducing an EMT-like phenotypic change and MMP-9-mediated degradation of E-cadherin into soluble E-cadherin (sE-cad) associated with activation of ERK-1/2 and PI3K signaling pathways (25). In the present study, similar results were obtained $(46,47)$. The results of the present study demonstrated that overexpression of CXCR4 can lead to the expression of EGFR and enhance the production of MMP-9, which finally results in elevating cell migration and invasion.

In conclusion, the results of the present study indicate that CXCR4 overexpression enhances cellular motility and invasion via EGFR and MMP-9 in NSCLC. It was also revealed 


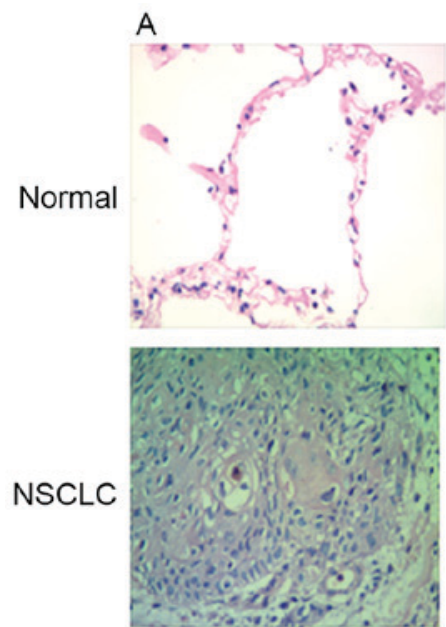

$H \& E$
B

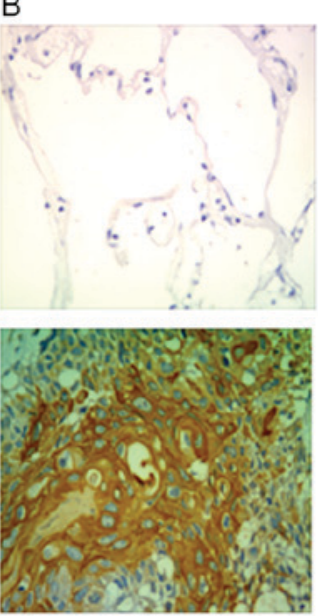

CXCR4
C
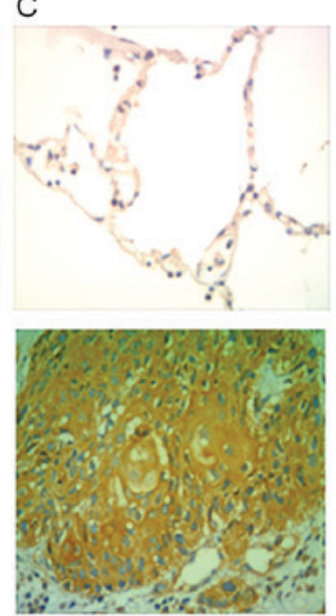

EGFR
D
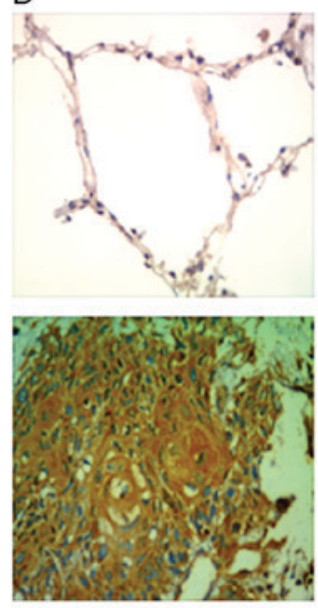

MMP-9

Figure 4. EGFR, CXCR4 and MMP-9 expression in NSCLC. (A) Hematoxylin and eosin staining is exhibited. (B) The expression of CXCR4, (C) EGFR and (D) MMP-9 in normal lung tissue and NSCLC tissue was examined using immunohistochemistry (magnification, x40). CXCR4, CXC receptor 4; EGFR, epidermal growth factor 4; MMP-9, matrix metallopeptidase-9; NSCLC, non-small cell lung cancer.

A

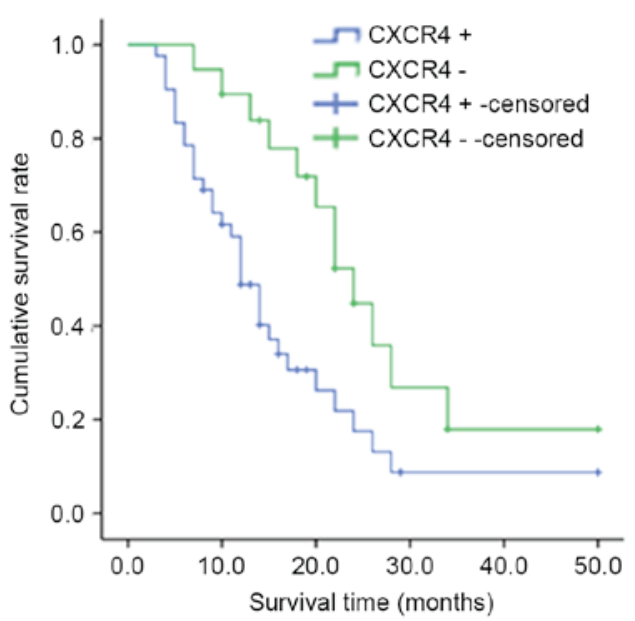

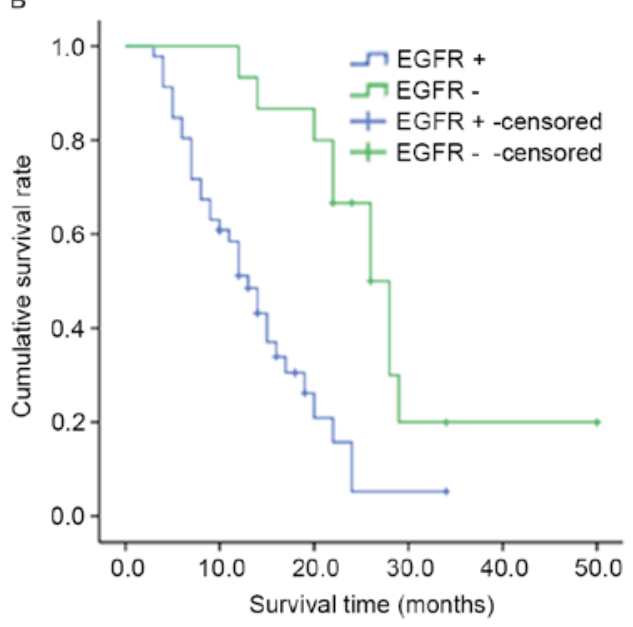

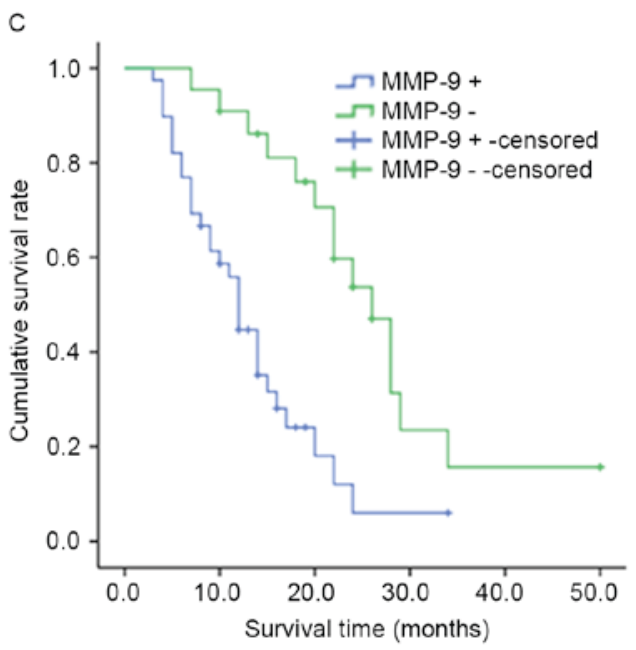

Figure 5. Kaplan-Meier estimates of overall survival according to the expression of (A) CXCR4, (B) EGFR and (C) MMP-9. CXCR4, CXC receptor 4; EGFR, epidermal growth factor 4; MMP-9, matrix metallopeptidase-9.

that the expression of CXCR4, EGFR and MMP-9 is associated with pathological grading and lymph node metastasis.
Furthermore, positive correlations were observed among CXCR4, EGFR and MMP-9 in NSCLC. 


\section{Acknowledgements}

The present study was supported by grants from the National Nature Science Foundation of China (grant no. 81272960), the Key Research Program from Science and Technology Department of Hunan Province, China (grant no. 2013WK2010), the Key Research Program from Ministry of Human Resources and Social Security of the People's Republic of China (grant no. 2016176), the Hunan Province Key Laboratory of Tumor Cellular and Molecular Pathology (grant no. 2016TP1015), the Construction Program of the Key Discipline in Hunan Province, China (Basic Medicine Sciences in University of South China, grant no. 201176), the General Research Program from Science and Technology Department of Hunan Province, China (grant no. 2013FJ3145), the Funds for the Development of Chemical Industry of Forest Products in Hunan Province Key Laboratory (grant no. JDLC201203) and the Wu Jieping Foundation Research Fund Project (grant no. 320.6750.12245).

\section{References}

1. Stewart BW and Wild CP (eds): World Cancer Report 2014. World Health Organization, IARC, Lyon, 2015.

2. He J and Song X: Advances in association of estrogen and human non-small cell lung cancer. Zhongguo Fei Ai Za Zhi 18: 315-320, 2015.

3. Schvartsman G, Ferrarotto R and Massarelli E: Checkpoint inhibitors in lung cancer: Latest developments and clinical potential. Ther Adv Med Oncol 8: 460-473, 2016.

4. Saavedra D and Crombet T: CIMAvax-EGF: A new therapeutic vaccine for advanced non-small cell lung cancer patients. Front Immunol 8: 269, 2017.

5. Singh AK, Arya RK, Trivedi AK, Sanyal S, Baral R, Dormond O, Briscoe DM and Datta D: Chemokine receptor trio: CXCR3, CXCR4 and CXCR7 crosstalk via CXCL11 and CXCL12. Cytokine Growth Factor Rev 24: 41-49, 2013.

6. Kodama J, Hasengaowa, Kusumoto T, Seki N, Matsuo T, Ojima Y, Nakamura K, Hongo A and Hiramatsu Y: Association of CXCR4 and CCR7 chemokine receptor expression and lymph node metastasis in human cervical cancer. Ann Oncol 18: 70-76, 2007.

7. Guo L, Cui ZM, Zhang J and Huang Y: Chemokine axes CXCL12/CXCR4 and CXCL16/CXCR6 correlate with lymph node metastasis in epithelial ovarian carcinoma. Chin J Cancer 30: 336-343, 2011.

8. Shen B, Zheng MQ, Lu JW, Jiang Q, Wang TH and Huang XE: CXCL12-CXCR4 promotes proliferation and invasion of pancreatic cancer cells. Asian Pac J Cancer Prev 14: 5403-5408, 2013.

9. Qiu MY, Li JW and Zheng MH: The chemokine receptors CXCR4 and CXCR7 in cancer. Chin Oncol 20: 222-226, 2010.

10. Kim M, Koh YJ, Kim KE, Koh BI, Nam DH, Alitalo K, Kim I and Koh GY: CXCR4 signaling regulates metastasis of chemoresistant melanoma cells by a lymphatic metastatic niche. Cancer Res 70: 10411-10421, 2010.

11. Maderna E, Salmaggi A, Calatozzolo C, Limido L and Pollo B: Nestin, PDGFRbeta, CXCL12 and VEGF in glioma patients: Different profiles of (pro-angiogenic) molecule expression are related with tumor grade and may provide prognostic information. Cancer Biol Ther 6: 1018-1024, 2007.

12. Driessen WH,FujiiN,TamamuraHandSullivanSM:Development of peptide-targeted lipoplexes to CXCR4-expressing rat glioma cells and rat proliferating endothelial cells. Mol Ther 16: 516-524, 2008.

13. Gulati S, Ytterhus B, Granli US, Gulati M, Lydersen S and Torp SH: Overexpression of c-erbB2 is a negative pognostic factor in anaplastic astrocytomas. Diagn Pathol 5: 18, 2010.

14. Lemmon MA and Schlessinger J: Cell signaling by receptor tyrosine kinases. Cell 141: 1117-1134, 2010.

15. Rexer BN, Ghosh R, Narasanna A, Estrada MV, Chakrabarty A, Song Y, Engelman JA and Arteaga CL: Human breast cancer cells harboring a gatekeeper T798M mutation in HER2 overexpress EGFR ligands and are sensitive to dual inhibition of EGFR and Her2. Clin Cancer Res 19: 5390-5401, 2013.
16. Al Zobair AA, Al Obeidy BF, Yang L, Yang C, Hui Y, Yu H, Zheng F, Yang G, Xie C, Zhou F, et al: Concomitant overexpression of EGFR and CXCR4 is associated with worse prognosis in a new molecular subtype of non-small cell lung cancer. Oncol Rep 29: 1524-1532, 2013.

17. Guo Z, Cai S, Fang R, Chen H, Du J, Tan Y, Ma W, Hu H, Cai S and Liu Y: The synergistic effects of CXCR4 and EGFR on promoting EGF-mediated metastasis in ovarian cancer cells. Colloids Surf B Biointerfaces 60: 1-6, 2007.

18. Patel LR, Camacho DF, Shiozawa Y, Pienta KJ and Taichman RS: Mechanisms of cancer cell metastasis to the bone: A multistep process. Future Oncol 7: 1285-1297, 2011.

19. Shon SK, Kim A, Kim JY, Kim KI, Yang Y and Lim JS: Bone morphogenetic protein- 4 induced by NDRG2 expression inhibits MMP-9 activity in breast cancer cells. Biochem Biophys Res Commun 385: 198-203, 2009.

20. Faraji SN, Mojtahedi Z, Ghalamfarsa G and Takhshid MA: $\mathrm{N}$-myc downstream regulated gene 2 overexpression reduces matrix metalloproteinase- 2 and -9 activities and cell invasion of A549 lung cancer cell line in vitro. Iran J Basic Med Sci 18: 773-779, 2015.

21. Zuo J, Ishikawa T, Boutros S, Xiao Z, Humtsoe JO and Kramer RH: Bcl-2 overexpression induces a partial epithelial to mesenchymal transition and promotes squamous carcinoma cell invasion and metastasis. Mol Cancer Res 8: 170-182, 2010.

22. Gao J, Liu X, Yang F, Liu T, Yan Q and Yang X: By inhibiting Ras/Raf/ERK and MMP-9, knockdown of EpCAM inhibits breast cancer cell growth and metastasis. Oncotarget 6: 27187-27198, 2015

23. Su L, Zhou W, Park S, Wain JC, Lynch TJ, Liu G and Christiani DC: Matrix metalloproteinase-1 promoter polymorphism and lung cancer risk. Cancer Epidemiol Biomarkers Prev 14: 567-570, 2005

24. Cai J, Li R, Xu X, Zhang L, Wu S, Yang T, Fang L, Wu J, Zhu X, Li M, et al: URGCP promotes non-small cell lung cancer invasiveness by activating the NF-kappaB-MMP-9 pathway. Oncotarget 6: 36489-36504, 2015.

25. Zuo JH, Zhu W, Li MY, Li XH, Yi H, Zeng GQ, Wan XX, He QY, Li JH, Qu JQ, et al: Activation of EGFR promotes squamous carcinoma SCC10A cell migration and invasion via inducing EMT-like phenotype change and MMP-9-mediated degradation of E-cadherin. J Cell Biochem 112: 2508-2517, 2011.

26. Travis WD: The 2015 WHO classification of lung tumors. Pathologe 35 (Suppl 2): S188, 2014.

27. Zuo J, Wen M, Lei M, Peng X, Yang X and Liu Z: MiR-210 links hypoxia with cell proliferation regulation in human Laryngocarcinoma cancer. J Cell Biochem 116: 1039-1049, 2015.

28. Dai X, Mao Z, Huang J, Xie S and Zhang H: The CXCL12/CXCR4 autocrine loop increases the metastatic potential of non-small cell lung cancer in vitro. Oncol Lett 5: 277-282, 2013.

29. Siegel R, Naishadham D and Jemal A: Cancer statistics, 2013. CA Cancer J Clin 63: 11-30, 2013.

30. Chang A: Chemotherapy, chemoresistance and the changing treatment landscape for NSCLC. Lung Cancer 71: 3-10, 2011

31. Zeng Z, Shi YX, Samudio IJ, Wang RY, Ling X, Frolova O, Levis M, Rubin JB, Negrin RR, Estey EH, et al: Targeting the leukemia microenvironment by CXCR4 inhibition overcomes resistance to kinase inhibitors and chemotherapy in AML. Blood 113: 6215-6224, 2009.

32. Peled A and Tavor S: Role of CXCR4 in the pathogenesis of acute myeloid leukemia. Theranostics 3: 34-39, 2013.

33. Sheikh Ali MA, Gunduz M, Nagatsuka H, Gunduz E, Cengiz B, Fukushima K, Beder LB, Demircan K, Fujii M, Yamanaka N, et al: Expression and mutation analysis of epidermal growth factor receptor in head and neck squamous cell carcinoma. Cancer Sci 99: 1589-1594, 2008.

34. Fung $\mathrm{C}$ and Grandis JR: Emerging drugs to treat squamous cell carcinomas of the head and neck. Expert Opin Emerg Drugs 15: 355-373, 2010.

35. Teicher BA and Fricker SP: CXCL12 (SDF-1)/CXCR4 pathway in cancer. Clin Cancer Res 16: 2927-2931, 2010.

36. Murakami T, Kawada K, Iwamoto M, Akagami M, Hida K, Nakanishi Y, Kanda K, Kawada M, Seno H, Taketo MM, et al: The role of CXCR3 and CXCR4 in colorectal cancer metastasis. Int J Cancer 132: 276-287, 2013.

37. Yang SX, Loo WT, Chow LW, Yang XH, Zhan Y, Fan LJ, Zhang F, Chen L, Wang QL, Xiao HL, et al: Decreased expression of C-erbB-2 and CXCR4 in breast cancer after primary chemotherapy. J Transl Med 10 (Suppl 1): S3, 2012. 
38. Ying J, Xu Q, Zhang G, Liu B and Zhu L: The expression of CXCL12 and CXCR4 in gastric cancer and their correlation to lymph node metastasis. Med Oncol 29: 1716-1722, 2012.

39. Billah S, Stewart J, Staerkel G, Chen S, Gong Y and Guo M: EGFR and KRAS mutations in lung carcinoma: Molecular testing by using cytology specimens. Cancer Cytopathol 119: 111-117, 2011.

40. Okamoto I: Epidermal growth factor in relation to tumor development: EGFR-targeted anticancer therapy. FEBS J 277: $309-315,2010$.

41. Lichtenberger BM, Tan PK, Niederleithner H, Ferrara N, Petzelbauer P and Sibilia M: Autocrine VEGF signaling synergizes with EFGR in tumor cells to promote epithelial cancer development. Cell 140: 268-279, 2010

42. Mitsudomi T and Yatabe Y: Epidermal growth factor receptor in relation to tumor development: EGFR gene and cancer. FEBS J 277: 301-308, 2010.

43. Gajiwala KS, Feng J, Ferre R, Ryan K, Brodsky O, Weinrich S, Kath JC and Stewart A: Insights into the aberrant activity of mutant EGFR kinase domain and drug recognition. Structure 21 209-219, 2013
44. Morrison LE, Jacobson KK, Friedman M, Schroeder JW and Coon JS: Aberrant EGFR and chromosome 7 associate with outcome in laryngeal cancer. Laryngoscope 115: 1212-1218, 2005.

45. Tzeng CD, Frolov A, Howard JH, Vickers SM, Buchsbaum DJ, Heslin MJ and Arnoletti JP: 96: Analysis of aberrant epidermal growth factor rector (EGFR) pathway signaling in pancreatic cancer patients: Implications for anti-EGFR therapy. J Surg Res 137: 191, 2007.

46. Shen B, Zheng MQ, Lu JW, Jiang Q, Wang TH and Huang XE: CXCL12-CXCR4 promotes proliferation and invasion of pancreatic cancer cells. Asian Pac J Cancer Prev 14: 5403-5408, 2013.

47. Pei J, Lou Y, Zhong R and Han B: MMP9 activation triggered by epidermal growth factor induced FoxO1 nuclear exclusion in non-small cell lung cancer. Tumour Biol 35: 6673-6678, 2014. 